\title{
Island Shape Transition in Heteroepitaxial Metal Growth on Square Lattices
}

\author{
Bert Müller,* Lorenz Nedelmann, Bjørn Fischer, Harald Brune, Johannes V. Barth, and Klaus Kern \\ Institut de Physique Expérimentale, Ecole Polytechnique Fédérale de Lausanne, CH-1015 Lausanne, Switzerland
}

(Received 3 February 1997)

\begin{abstract}
A novel mechanism for ramified island growth in the initial stages of metal heteroepitaxy is reported. Scanning tunneling microscopy measurements reveal that copper islands on $\mathrm{Ni}(100)$, as they grow in size, undergo a shape transition. Below a critical size of $\approx 480$ atoms, compact islands form, while above this size they develop a ramified shape. This effect is not of kinetic origin and has been observed in an extended range of growth temperature $(250-370 \mathrm{~K})$ and deposition flux $\left(10^{-5}-10^{-2}\right.$ monolayer/s). The shape transition is ascribed to the island size dependent strain relaxation. [S0031-9007(98)05561-6]
\end{abstract}

PACS numbers: 68.35.Bs, 61.16.Ch, 68.55.Jk

The understanding of the initial stages of epitaxial growth on metal surfaces has progressed rapidly over the past few years. In particular, scanning tunneling microscopy (STM) has provided important contributions, unraveling, e.g., the atomic mechanisms of phenomena such as fractal or dendritic growth [1-5]. The ramified island shapes observed at low growth temperature are of kinetic origin, determined by the competition between the lateral impingement rate to an island (determined by terrace diffusion and deposition flux) and the edge and corner diffusion after the atom is attached to a growing island. Substrate symmetry is expected to play a decisive role in the formation of the observed island morphologies [6]. Ramified islands with dendritic or fractal shape are expected only on triangular or hexagonal but not on square lattices. The difference in shape is explained by the higher barrier of edge diffusion on triangular lattices due to the presence of twofold coordinated edge sites. Thus, on square lattices, fabrication of ramified islands should be possible only at very low growth temperatures, or be even inhibited for systems where the barrier of edge diffusion is lower than that of terrace diffusion $[7,8]$. This picture is consistent with experimental observations; so far exclusively compact islands have been observed in metal epitaxy on substrates with square symmetry [9-14].

It is the aim of the present Letter to demonstrate that ramified islands can also grow on substrates with square symmetry. The physical mechanism responsible for island ramification is, however, related to the strain relief in heteroepitaxial islands. We present STM data showing a shape transition of copper islands on $\mathrm{Ni}(100)$ at submonolayer coverages over a wide temperature range $(250-370 \mathrm{~K})$ at usual growth conditions. While small islands exhibit the expected compact shape, islands exceeding a critical size of $\approx 480$ atoms are ramified. Because of the elevated growth temperatures and the invariance of the transition on temperature and flux, the island shape is not of kinetic origin. The shape transition results from the compressive strain in the copper islands due to the positive lattice mismatch of the two metals $(+2.58 \%)$. Upon ramification the islands increase their perimeter, where strain can efficiently be relieved through outward relaxation of the edge atoms.

The experiments were performed with a variable temperature STM, incorporated in a standard ultrahigh vacuum chamber; analogous instrumentation has been described elsewhere [15]. The $\mathrm{Ni}(100)$ crystal was prepared by cycles of argon ion sputtering $\left(1 \mu \mathrm{A} / \mathrm{cm}^{2}\right)$ and subsequent annealing $(1200 \mathrm{~K})$ resulting in defect-free terraces of $\sim 1000 \AA$ width. Copper was deposited by thermal evaporation from a Knudsen-type source at background pressures below $1 \times 10^{-9}$ mbar. The deposition rate was varied between $3 \times 10^{-5}$ and $3 \times 10^{-2} \mathrm{ML} / \mathrm{s}$ and calibrated from STM images at monolayer (ML) coverage. The STM measurements were performed in the constant current mode at $0.5-2.0 \mathrm{~V}$ positive or negative bias and 0.5-8.0 nA tunneling current.

A series of STM images revealing the evolution of the $\mathrm{Cu}$ island shapes with increasing coverage at a substrate temperature of $345 \mathrm{~K}$ is reproduced in Fig. 1 . From the beginning $\mathrm{Cu}$ grows in two-dimensional islands of monolayer height. At very low coverage there are exclusively small islands with compact shape. As the size of the islands increases with $\mathrm{Cu}$ coverage, their shape becomes irregular above a critical size $A_{c}$ of about 480 atoms. For $\Theta \geq 0.1 \mathrm{ML}$ the majority of the islands is ramified and their arms exhibit a preferential width of about 20 atoms. Note that the branches are oriented preferentially parallel and perpendicular to the substrate step edges; i.e., they run along close-packed directions. This growth scenario is characteristic for a wide temperature range (250-370 K) and independent of the $\mathrm{Cu}$ flux, as verified for the entire flux range. The only effect of both change of substrate temperature and deposition flux is a change in island density, but the critical island size of about 480 atoms, where ramification sets in, remains unaffected. The comparison of the data in Fig. 1 (growth temperature $345 \mathrm{~K}$ ) and Fig. 2 (growth temperature $250 \mathrm{~K}$ ) reveals similar island shapes for similar mean island sizes [the mean island sizes are $S \approx 100, \approx 1200(\approx 80, \approx 1000)$ atoms for a coverage of $0.02,0.15(0.09,0.50)$ ML grown at $T=345(250) \mathrm{K}$, 

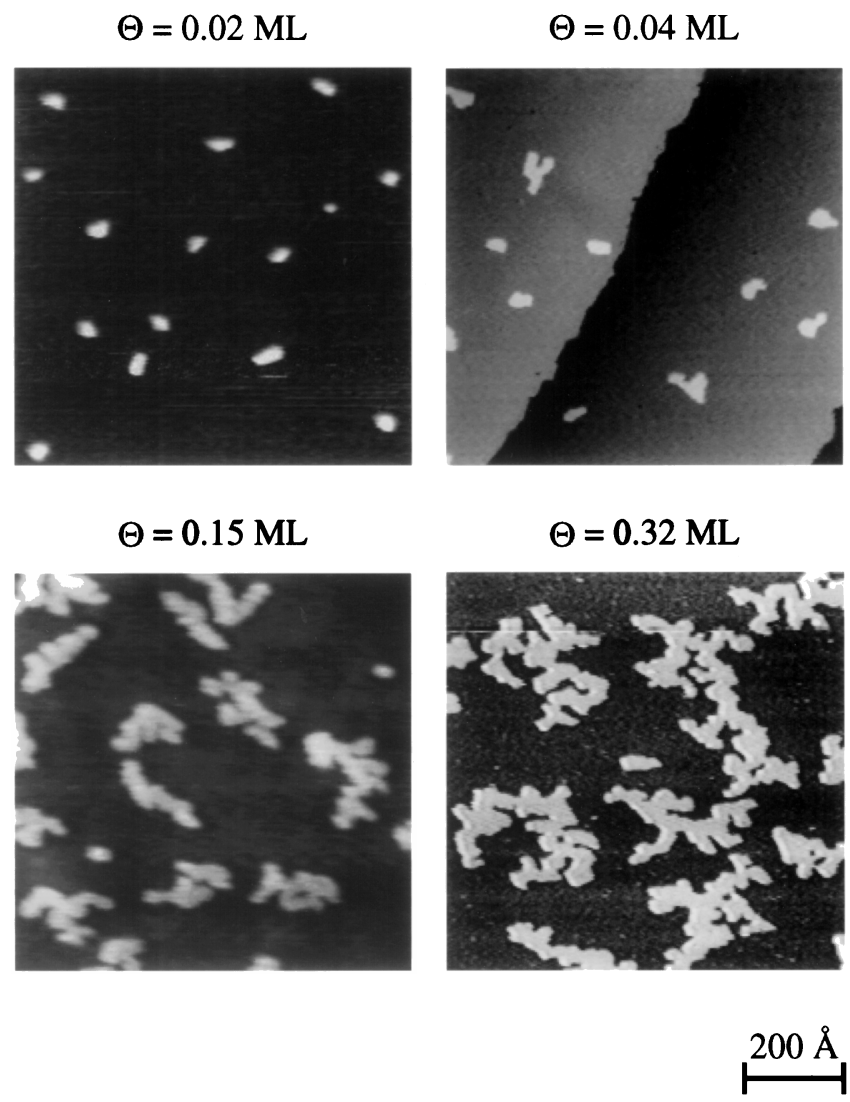

FIG. 1. Submonolayer growth of copper islands on Ni(100) characterized by a transition from compact to ramified island shapes with increasing island size. The islands are grown at a substrate temperature of $345 \mathrm{~K}$ and a growth rate of $1.5 \times 10^{-3} \mathrm{ML} / \mathrm{s}$. Coverages as indicated.

respectively]. In order to gain insight into the physics behind the island shape transition, the topography of more than 3000 islands has been analyzed. The evaluation of the island perimeter $(p)$ vs island size $(A)$ is displayed as a double logarithmic plot in Fig. 3. In the upper part of the figure, the black dots corresponding to individual islands represent the raw data, while the colored dots show averaged data color coded according to the growth temperature. In the lower part the data for $350 \mathrm{~K}$ are further analyzed as a function of deposition flux. This representation clearly demonstrates that the shape transition is independent of both growth temperature and deposition flux.

In view of these results and the high mobility of the adsorbed $\mathrm{Cu}$ atoms at the employed temperature range (average terrace diffusion length of several hundred $\AA$ ), which even includes the transition from a critical nucleus of $i=1$ to $i=3$ [16], it can be safely concluded that the observed island shapes are not of kinetic origin. Hence, the growth kinetics do not determine the island shape transition and for a quantitative analysis of this phenomenon all data taken at various growth conditions can be used. In all cases for island sizes up to $\approx 300$ atoms compact islands are formed, whose perime- ter scales with the square root of the island size. For larger islands a deviation from this behavior is observed, and the dependence cannot be described in simple analytical terms, until for island sizes exceeding $\approx 3000$ atoms the island perimeter is found to be directly proportional to the area. The observed behavior can be modeled by the growth of a linear chain with a certain arm width $w$ (orange line). The arm width is obtained by fitting the data for islands exceeding the critical size using $p=$ $2 A / w+2 w$, where the arm width $w$ is the only fit parameter. The optimum fit value of the arm width $w=22 \pm$ 1 is quite independent of the starting point. The corresponding critical island size is $A_{c}=480 \pm 20$ atoms.

The driving force for this preferential arm width and thus for the ramification of the $\mathrm{Cu}$ islands on the $\mathrm{Ni}(100)$ substrate is associated with the lattice mismatch of the two materials. The effect of strain is usually not considered in simple diffusion limited growth models, which accordingly fail to provide a realistic description of the present system. Particularly important is the sign of the lattice misfit as demonstrated by the fact that copper forms ramified islands on $\mathrm{Ni}(100)$ but not on $\mathrm{Pd}(100)$ [11]. Both substrate materials are very similar. The copper islands, however, are compressively strained on $\mathrm{Ni}(100)$ and exhibit tensile strain on $\operatorname{Pd}(100)$ due to the positive and negative misfit, respectively.

Since the step edge atoms are bound only to one side and therefore free to relax outwards they follow their natural lattice spacing which is larger than that of the substrate. On the other hand, the lower coordination of the edge atoms favors inward relaxation since less coordination tends to shrink bond lengths. In general, these two effects compete and it is a priori difficult to determine the dominant term. For the particular case of $\mathrm{Cu} / \mathrm{Ni}(100)$ we have performed calculations using effective medium theory (EMT) [7] which reveal indeed an outward relaxation of the edge atoms, confirming the dominance of strain effects. The importance of strain effects for this system can also be inferred from strain
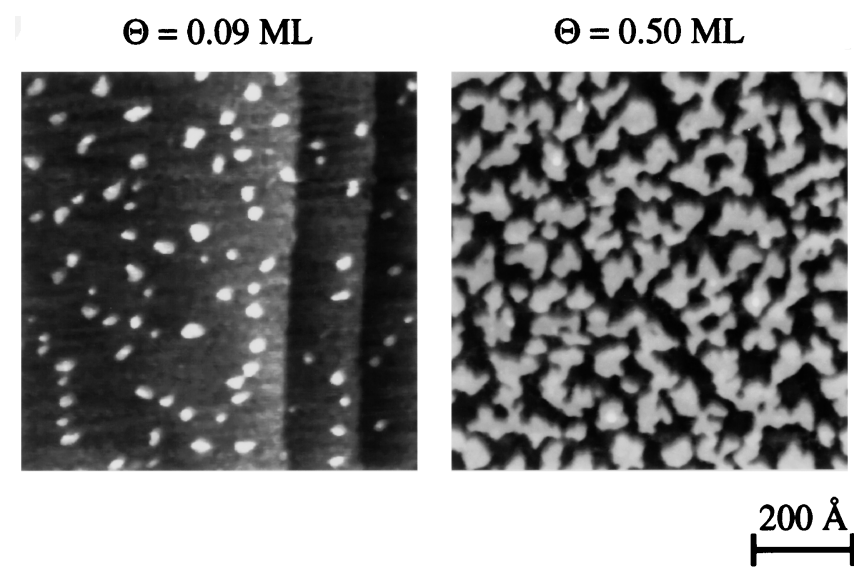

FIG. 2. Island ramification for a substrate temperature of $250 \mathrm{~K}$ (deposition flux $1.5 \times 10^{-3} \mathrm{ML} / \mathrm{s}$; note the higher island density than in Fig. 1 due to the reduced temperature). 


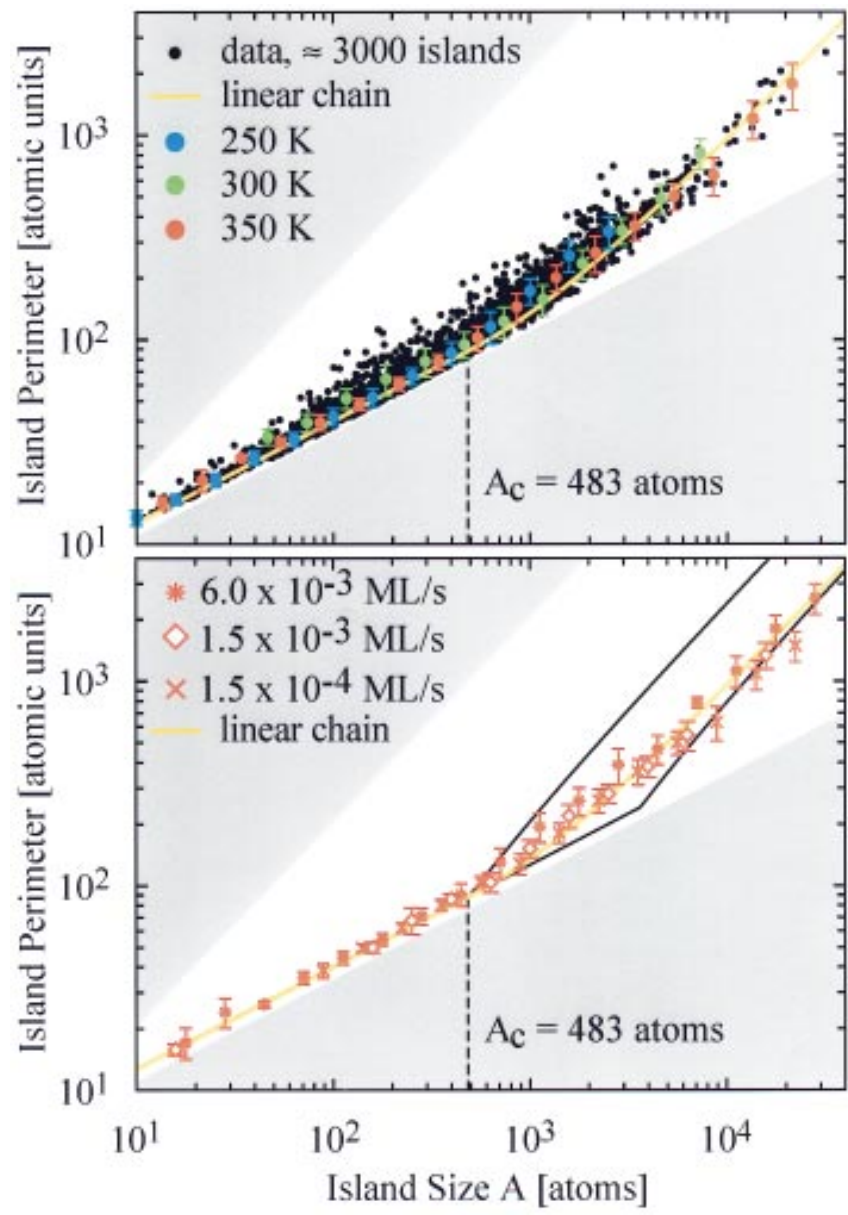

FIG. 3(color). Island perimeter $p$ vs island size $A$ for submonolayer copper islands on $\mathrm{Ni}(100)$ obtained at different growth temperatures $(T=250,300$, and $350 \mathrm{~K})$, growth rates $\left(3 \times 10^{-5}-3 \times 10^{-2} \mathrm{ML} / \mathrm{s}\right)$, and coverages (0.06-0.66 ML), characterizing the size-dependent shape transition. The upper part shows the data of more than 3000 islands, each dot corresponding to one island (the grey shaded area is the forbidden region for the ratios; its upper limit corresponds to that of linear atomic chains, the lower limit to circular islands). The colored dots show averaged data which are displayed with their statistical error bars and are color coded according to the growth temperature (blue: $250 \mathrm{~K}$; green: $300 \mathrm{~K}$; red: $350 \mathrm{~K}$ ). In the lower part, the $350 \mathrm{~K}$ data are further analyzed as a function of deposition flux (stars: $6.0 \times 10^{-3} \mathrm{ML} / \mathrm{s}$, rhombi: $1.5 \times 10^{-3} \mathrm{ML} / \mathrm{s}$, crosses: $\left.1.5 \times 10^{-4} \mathrm{ML} / \mathrm{s}\right)$. The data are compared with the linear chain model: $p=2 A / 22+2 \times 22$ (orange line) and the model from Ref. [19] with $b=1.1$ and $b=2.1$ (black lines).

relaxation via "internal faceting" which has been observed for larger $\mathrm{Cu}$ islands [17].

The fact that the ramification of the islands involves preferential growth along close-packed directions indicates that (i) there is enough mobility along the edge and (ii) kink sites are energetically unfavorable. Both indications are corroborated by EMT results. As for other square lattices the barrier for edge diffusion $(285 \mathrm{meV})$ is found to be lower than for terrace diffusion $(469 \mathrm{meV})$ and the barrier for corner diffusion $(530 \mathrm{meV})$ is only slightly larger. In addition, we have found that there is no effect of the island size on the activation barrier for edge and corner diffusion. Kink sites are energetically costly since they reduce coordination while leaving the number of edge atoms constant. The observation of a constant arm width also implies that growth becomes anisotropic and atoms attaching sideways diffuse towards the tip. The optimum arm width of $\sim 22$ atoms is reasonable since it is well below the critical island size for the onset of strain relaxation via internal $\{111\}$ faceting $[17,18]$.

The ramified island shape can be understood as a result of the energy balance of the atomic bond energy within the islands and the strain energy due to the lattice mismatch with the substrate. Therefore, one can estimate an upper limit for the strain energy by the determination of the bond energy difference between the observed ramified islands and square islands of identical size. Based on bond counting, the binding energy of linear chain islands corresponds to $(2 A-p / 2) E_{b}$, where $A$ and $p$ are expressed in the number of atoms forming the island and its perimeter, respectively. For a square island, $p=4 \sqrt{A}$, and the bond energy per atom is given by $(2-2 / \sqrt{A}) E_{b}$. For the ramified islands, however, we have found $p=A / 11+44$, which results in a bond energy per atom of $\left(2-\frac{1}{22}-22 / A\right) E_{b}$. Hence, for very large islands, the energy gain $E^{*}$ is $\frac{1}{22}$ of the dimer bond energy $E_{b}$ per atom. Using the value for the dimer bond energy of Ref. [16] $E_{b}=(0.46 \pm 0.19) \mathrm{eV}$, the energy gain $E^{*}$ corresponds to $(21 \pm 9) \mathrm{meV}$ per island atom. This value, which is comparable to strain energies calculated from bulk properties, is relatively small and it is reasonable to assume that the difference can be overbalanced by the energy gain associated with the more effective strain relief at the longer edges of ramified islands. On the other hand, it is high enough to explain that the arm width is constant over the wide temperature range between 250 and $370 \mathrm{~K}$.

A strain induced spontaneous shape transition in the growth of heteroepitaxial islands has been theoretically predicted by Tersoff and Tromp [19]. They considered three-dimensional islands of rectangular shape with width $s$, length $t$, and height $h$ and determined their excess surface and strain energy to $E=b(s+t)-s \ln (t)-t \ln (s)$. If $b$ is considered to be constant, it is the only fit parameter in the model. Using this ansatz, the critical island size is given by $A_{c}=\exp (2 b+4)$ with a width of $s_{c}=\exp (b+2)$, whose asymptotic limit is $s_{\infty}=\exp (b+1)$ for very large islands. While only the simple rectangular shape was investigated, these results should apply equally well to the ramified shape, particularly if the arm length substantially exceeds its width. The branching does not affect the perimeter for a given island size. The obtained ratio between $p$ and $A$ is shown in the lower part of Fig. 3 for $b=1.1$ and $b=2.1$ by the black curve. An increase of $b$ only shifts the transition to higher island sizes. $A_{c}=480$ atoms results in $b=1.1$ and, therefore, $s_{c}=22$ atoms and $s_{\infty}=8$ atoms, whereby 
$b=2.1$ yields $A_{c}=3640$ atoms, $s_{c}=60$ atoms, and $s_{\infty}=22$ atoms for infinite island sizes. We do not find this shrinking, which might be related to the limited island size, experimentally accessible. In addition the model predicts a sharp transition between two slopes, whereas we find a continuous one. Hence there is only qualitative agreement [20]. Nevertheless, the theory correctly predicts the spontaneous shape transition in growth of monolayer-high islands due to lattice strain and our investigation is the first direct verification of this theoretical prediction.

The mechanism of relaxation driven island shape transition is further substantiated by additional STM observations, where the surface was annealed above the critical temperature for surface alloy formation $(T>400 \mathrm{~K})$. The data presented in Fig. 4 demonstrate that upon intermixing of the substrate and island materials compact islands form again. (For this experiment a temperature was chosen, where the islands are not yet dissolved, but exchange processes between $\mathrm{Cu}$ islands and the Ni substrate occur.) The effect of intermixing can be easiest seen at the step edges, which are straightened and whose rims are imaged higher and spotted. The rims are not well separated from the Ni substrate and a rather irregular interface is formed. The islands are imaged with the same height and exhibit a similar spotted surface and hence consist also of randomly mixed $\mathrm{Ni}$ and $\mathrm{Cu}$. Since the incorporated $\mathrm{Ni}$ atoms are smaller, the strain energy of the islands is significantly reduced [21] and consequently compact islands form (comprising up to $\approx 1000$ atoms in the data shown).

In conclusion, we have observed a shape transition of copper islands on $\mathrm{Ni}(100)$ from compact to ramified islands with a critical island size of $\approx 480$ atoms. The ramification of larger islands is ascribed to anisotropic strain relief at island edges. The experimental observation of the shape transition lends support to earlier theoretical predictions of Tersoff and Tromp [19]. This phenomenon is expected to be of general importance in heteroepitaxy on square
Growth at $345 \mathrm{~K}$

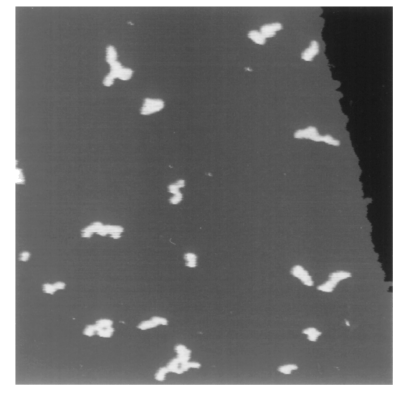

Annealed to $450 \mathrm{~K}$

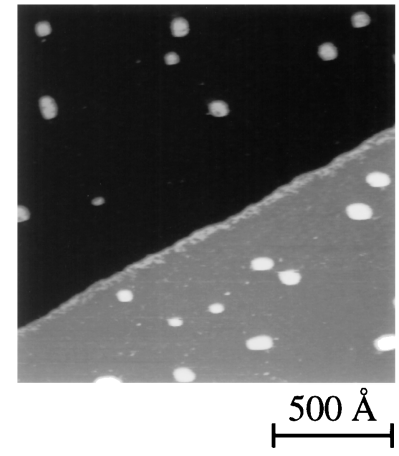

FIG. 4. STM images of copper islands on $\mathrm{Ni}(100)$ before and after annealing to $450 \mathrm{~K}$ (growth temperature $345 \mathrm{~K}$, deposition rate $6 \times 10^{-4} \mathrm{ML} / \mathrm{s}$, coverage $0.04 \mathrm{ML}$ ), demonstrating the effect of surface alloying on the island and step shape. lattices with a positive misfit of the materials and shows the possible drastic influence of strain on island shapes.

It is our pleasure to thank Jerry Tersoff for pointing out the relevance of Ref. [19] and stimulating discussions concerning the application of the analytic strain theory in the present investigation. Likewise, fruitful discussions with Karsten Bromann and financial support from the Alexander von Humboldt-Stiftung (B.M.) and the Deutscher Akademischer Austauschdienst (L. N.) are gratefully acknowledged.

*Present address: Institut für Quantenelektronik, Eidgenössische Technische Hochschule Zürich, CH-8093 Zürich, Switzerland.

[1] R. Hwang, J. Schröder, C. Günther, and R. J. Behm, Phys. Rev. Lett. 67, 3297 (1991).

[2] H. Brune, C. Romainczyk, H. Röder, and K. Kern, Nature (London) 369, 469 (1994).

[3] H. Röder, K. Bromann, H. Brune, and K. Kern, Phys. Rev. Lett. 74, 3217 (1995).

[4] M. Hohage, M. Bott, M. Morgenstern, Z. Zhang, T. Michely, and G. Comsa, Phys. Rev. Lett. 76, 2366 (1996).

[5] H. Brune, K. Bromann, J. Jacobsen, K. Jacobsen, P. Stoltze, J. Nørskov, and K. Kern, Surf. Sci. Lett. 349, L115 (1996).

[6] Z. Zhang, X. Chen, and M. G. Lagally, Phys. Rev. Lett. 73, 1829 (1994).

[7] P. Stoltze, J. Phys. Condens. Matter 6, 9495 (1994).

[8] C.-L. Liu, Surf. Sci. 316, 294 (1994).

[9] E. Kopatzki, S. Günther, W. Nichtl-Pecher, and R. J. Behm, Surf. Sci. 284, 154 (1993).

[10] J. A. Stroscio, D. T. Pierce, and R. A. Dragoset, Phys. Rev. Lett. 70, 3615 (1995).

[11] E. Hahn, E. Kampshoff, N. Wälchli, and K. Kern, Phys. Rev. Lett. 74, 1803 (1995).

[12] D. D. Chambliss and K. E. Johnson, Phys. Rev. B 50, 5012 (1994).

[13] J. M. Wen, J. W. Evans, M. C. Bartelt, J. W. Burnett, and P. A. Thiel, Phys. Rev. Lett. 76, 652 (1996).

[14] The only exception where irregular islands were reported on a square lattice is the case of $\mathrm{Ag} / \mathrm{Ni}(100)$, which grows in hexagonal symmetry. A. Brodde, G. Wilhelm, D. Badt, H. Wengelnik, and H. Neddermeyer, J. Vac. Sci. Technol. B 9, 920 (1991).

[15] H. Brune, H. Röder, K. Bromann, and K. Kern, Thin Solid Films 264, 230 (1995).

[16] B. Müller, B. Fischer, L. Nedelmann, H. Brune, and K. Kern, Phys. Rev. B 54, 17858 (1996).

[17] B. Müller, B. Fischer, L. Nedelmann, A. Fricke, and K. Kern, Phys. Rev. Lett. 76, 2358 (1996).

[18] B. Müller, L. Nedelmann, B. Fischer, A. Fricke, and K. Kern, J. Vac. Sci. Technol. A 14, 1878 (1996).

[19] J. Tersoff and R. M. Tromp, Phys. Rev. Lett. 70, 2782 (1993).

[20] B. Müller, L. Nedelmann, B. Fischer, H. Brune, J. V. Barth, K. Kern, D. Erdös, and J. Wollschläger, Surf. Rev. Lett. (to be published).

[21] J. Tersoff, Phys. Rev. Lett. 74, 434 (1995). 